\title{
Phase Transition Behaviours of the Spontaneous Polarization in Ferroelectric Thin Films
}

\author{
Li Rong \\ Archives \\ Linyi University \\ Linyi 276005, China \\ e-mail: ruoshuilu@163.com
}

\begin{abstract}
Within the framework of the effective-field theory with correlations, the phase transition behaviors of the spontaneous polarization in a ferroelectric thin film with symmetrical structure have been studied in detail by the transverse field Ising model. A general expression for the phase diagram of the thin film with arbitrary exchange interactions and transverse fields is deduced through mathematical derivation. The four possible phase transition regions in the phase diagram and the Curie temperature dependence of spontaneous polarization are numerically calculated. It shows that both the spontaneous polarization and the Curie temperature can change in a different or same direction. The results are consistent with those of the usual mean-field approximation, but deviate from the Landau phenomenological theory. In addition, the temperature dependence of the spontaneous polarization in experimental facts of triglycine sulfate (TGS) thin films is also compared by the effective-field theory with correlations.
\end{abstract}

Keywords-ferroelectric thin film; transverse Ising model; phase diagram; spontaneous polarization; Curie temperature

\section{INTRODUCTION}

The surface and size effects on phase transition properties in various ferroelectrics have been extensively studied from experiment and theory since they were found in the 1950's [1]. With the development of artificial preparation technology in ferroelectric multilayer films and composites, some oxide thin films with a perovskite-type structure, such as $\mathrm{PbTiO}_{3} / \mathrm{SrTiO}_{3}(\mathrm{PT} / \mathrm{ST}), \mathrm{BaTiO}_{3} / \mathrm{SrTiO}_{3}$ (BT/ST), $\mathrm{SrZrO}_{3} / \mathrm{SrTiO}_{3}, \mathrm{~Pb}\left(\mathrm{Zr}_{0.8} \mathrm{Ti}_{0.2}\right) \mathrm{O}_{3} / \mathrm{Pb}\left(\mathrm{Zr}_{0.2} \mathrm{Ti}_{0.8}\right) \mathrm{O}_{3}$, and $\mathrm{ST} / \mathrm{BT} / \mathrm{CaTiO}_{3}$, have been successfully fabricated and have revealed some novel physics characteristics [2]. It has been demonstrated that the Curie temperature and the spontaneous polarization can be obviously changed by a modification on the surface layer in ferroelectric materials [3]. Hadni et al. [4] found that the Curie point of the triglycine sulfate (TGS) is significantly increased while the spontaneous polarization at a low temperature is reduced. Theoretically, the Landau phenomenological theory and the transverse field Ising model have been used to study the surface and size effects on ferroelectric phase transitions [5-]. As far as we know, the transverse field Ising model has been more fruitful and has yielded more good results. Wang et al. [6] and Sy [7] as well as Wang et al. [8] paid attention to the Curie temperature, respectively, by using the usual mean-field approximation. Wang et al. [9] studied three phase transition regions in the phase diagram. Qu et al. [3] found four possible regions and discussed the temperature dependence of spontaneous polarization. The results indicate that the spontaneous polarization and the Curie temperature might increase or decrease in a different direction. As far as we know, however, the investigation of four possible phase transition regions and the temperature dependence of the spontaneous polarization in [3] have not been carried out by the effective-field theory with correlations.

In this paper, the phase transition behaviors of spontaneous polarization in a ferroelectric thin film with symmetrical structure have been systematically investigated by the effective-field theory with correlations It is assumed that exchange interactions and transverse fields in symmetrical surface layers are different from those of bulk layers. Firstly the general expression for phase diagrams of a ferroelectric thin film with arbitrary interactions parameters is derived. Then the four possible phase transition regions in $\Omega_{s} / \Omega_{b} \sim J_{s} / J_{b}$ plane and the temperature dependence of spontaneous polarization are numerically examined. The results indicate that both the spontaneous polarization and the Curie temperature can be altered in a different or same direction. Meanwhile the temperature dependence of the spontaneous polarization for experimental facts of triglycine sulfate (TGS) thin films can also be described by the effective-field theory with correlations.

\section{THE MODEL AND FORMULATIONS}

The physical model of the ferroelectric thin film we considered in this paper is shown in Fig .1. It is assumed that the film has a three-dimensional simple cubic lattice symmetrical structure. The index of each layer is labeled as $1,2,3, \ldots, N$, respectively. Each layer is defined in the $x-y$ plane and the pseudo-spin sites on the square lattice. The film was supposed to have two surface layers (i.e., the top layer and the bottom layer numbered 1 and $N$ ), while the other $N-2$ layers are named as the bulk layers. The Hamiltonian for the ferroelectric system can be give as follows: [10] 


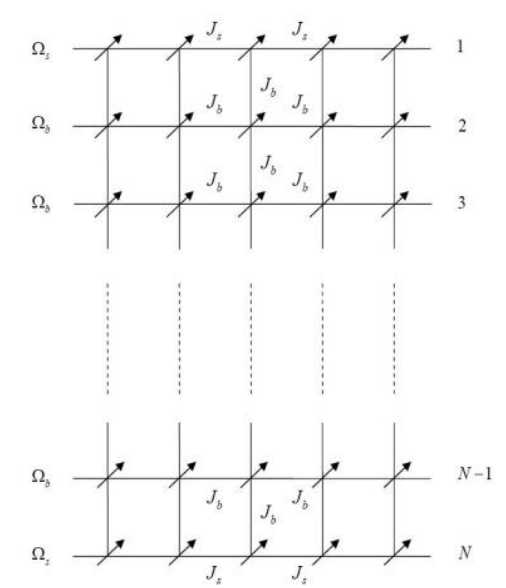

Figure 1. Schematic illustration of an $N$-layer ferroelectric thin film.

$$
H=-\frac{1}{2} \sum_{i j} J_{i j} S_{i}^{z} S_{j}^{z}-\Omega_{b} \sum_{i \in b} S_{i}^{x}-\Omega_{s} \sum_{i \in S} S_{i}^{x}
$$

where $J_{i j}$ is the nearest neighbor exchange interaction between sites $i$ and $j \cdot J_{i j}=J_{s}$ (or $J_{b}$ ) is the exchange interaction in the surface (or bulk) layers. Similarly, $\Omega_{s}$ (or $\Omega_{b}$ ) is the transverse field in the surface (or bulk) layers. $S_{i}^{x}$ and $S_{i}^{z}$ are the components of the pseudospin operator in $x$ and $z$ direction at site $i$.

Within the effective-field theory with correlations, the spontaneous polarization average in the $i$ th layer of ferroelectric thin film is [10]

$$
R_{i}=\left\langle S_{i}^{z}\right\rangle=\left.\left\langle\prod_{\delta}\left[\cosh \left(D J_{i, j+\delta}\right)+R_{j+\delta} \sinh \left(D J_{i, j+\delta}\right)\right]\right\rangle f_{i}(x)\right|_{x=0},
$$

where $\Pi$ is the cumprod for the nearest neighbor of site $i$, $D=\partial / \partial x$ is the differential operator and $\exp (\alpha D) f(x)=f(x+\alpha)$. In the top and bottom surface layers, the function $f_{i}(x)$ is defined as

$$
f_{i}(x)=f_{s}(x)=\frac{x}{\sqrt{\left(2 \Omega_{s}\right)^{2}+x^{2}}} \tanh \left[\frac{\beta}{4} \sqrt{\left(2 \Omega_{s}\right)^{2}+x^{2}}\right],
$$

whereas in the bulk layers, the function $f_{i}(x)$ is

$$
f_{i}(x)=f_{b}(x)=\frac{x}{\sqrt{\left(2 \Omega_{b}\right)^{2}+x^{2}}} \tanh \left[\frac{\beta}{4} \sqrt{\left(2 \Omega_{b}\right)^{2}+x^{2}}\right],
$$

where $\beta=1 /\left(k_{B} T\right), T$ is the temperature.

The spontaneous polarization average in the $i$ th layer of a ferroelectric thin film can be obtained from (2) as follows

$$
\begin{aligned}
R_{1}= & {\left[\cosh \left(D J_{s}\right)+R_{1} \sinh \left(D J_{s}\right)\right]^{4}, } \\
& \times\left.\left[\cosh \left(D J_{s}\right)+R_{2} \sinh \left(D J_{s}\right)\right] f_{s}(x)\right|_{x=0} \\
R_{2}= & {\left[\cosh \left(D J_{b}\right)+R_{1} \sinh \left(D J_{b}\right)\right], } \\
& \times\left[\cosh \left(D J_{b}\right)+R_{2} \sinh \left(D J_{b}\right)\right]^{4} \\
& \times\left.\left[\cosh \left(D J_{b}\right)+R_{3} \sinh \left(D J_{b}\right)\right] f_{b}(x)\right|_{x=0}
\end{aligned}
$$

$$
\begin{aligned}
R_{i}= & {\left[\cosh \left(D J_{b}\right)+R_{i-1} \sinh \left(D J_{b}\right)\right] } \\
& \times\left[\cosh \left(D J_{b}\right)+R_{i} \sinh \left(D J_{b}\right)\right]^{4} \\
& \times\left.\left[\cosh \left(D J_{b}\right)+R_{i+1} \sinh \left(D J_{b}\right)\right] f_{b}(x)\right|_{x=0}
\end{aligned}
$$

M

$$
\begin{aligned}
R_{N-1} & =\left[\cosh \left(D J_{b}\right)+R_{N-2} \sinh \left(D J_{b}\right)\right] \\
& \times\left[\cosh \left(D J_{b}\right)+R_{N-1} \sinh \left(D J_{b}\right)\right]^{4}, \\
& \times\left.\left[\cosh \left(D J_{b}\right)+R_{N} \sinh \left(D J_{b}\right)\right] f_{b}(x)\right|_{x=0} \\
R_{N}= & {\left[\cosh \left(D J_{b}\right)+R_{N-1} \sinh \left(D J_{b}\right)\right] } \\
& \times\left.\left[\cosh \left(D J_{s}\right)+R_{N} \sinh \left(D J_{s}\right)\right]^{4} f_{s}(x)\right|_{x=0}
\end{aligned}
$$

In order to obtain the phase diagram of a ferroelectric thin film, it is necessary that the above $N$ equations should be expanded. Meanwhile, we should notice the symmetrical structure of the thin film; therefore $R_{1}=R_{N}$, $R_{2}=R_{N-1}, R_{3}=R_{N-2}, \mathrm{~L}$. Thus the amount of the numerical calculation can be decreased greatly. When the temperature closes to the Curie temperature (i.e., $T->T_{c}$ ), $R_{i}$ is a small value. The higher-order terms of $R_{i}$ will tend to zero faster than the linear terms of $R_{i}$. As a result, we can neglect the higher-order terms and only consider the linear terms. Then the recursive determinant equation for the phase diagram of an $N$-layer ferroelectric thin film is determined by $[7,11]$

$$
\begin{aligned}
& \left|\begin{array}{ccccccc}
\frac{1-4 k_{1}}{k} & -\frac{k_{2}}{k} & & & & & \\
-1 & \frac{1}{k}-4 & -1 & & & & \\
& -1 & \frac{1}{k}-4 & & & & \\
& & & 0 & & & \\
& & & & \frac{1}{k}-4 & -1 & \\
& & & & -1 & \frac{1}{k}-4 & -1 \\
& & & & & -\frac{k_{2}}{k} & \frac{1-4 k_{1}}{k}
\end{array}\right|_{N \times N}=0 \\
& \begin{array}{c}
k_{1}=\left.\cosh ^{3}\left(D J_{s}\right) \cosh \left(D J_{b}\right) \sinh \left(D J_{s}\right) f_{s}(x)\right|_{x=0}, \\
k_{2}=\left.\cosh ^{4}\left(D J_{s}\right) \sinh \left(D J_{b}\right) f_{s}(x)\right|_{x=0}, \\
k=\left.\cosh ^{5}\left(D J_{b}\right) \sinh \left(D J_{b}\right) f_{b}(x)\right|_{x=0},
\end{array}
\end{aligned}
$$$$
\text { with }
$$

where $k_{1}, k_{2}$, and $k$ are the functions of temperature as shown in (3) and (4). According to formula of the determinant, a general expression for the phase diagram of a thin film can be obtained

$$
\left(4 k_{1}-1\right)^{2} B_{N-2}(\phi)+2 k_{2}\left(4 k_{1}-1\right) B_{N-3}(\phi)+k_{2}^{2} B_{N-4}(\phi)=0,
$$

where

$$
B_{N}(\phi)=\frac{\sinh [(N+1) \phi]}{\sinh (\phi)}, \cosh (\phi)=\frac{\frac{1}{k}-4}{2} .
$$




\section{NUMERICAL RESULTS}

In this section, we will investigate the surface modification on phase transition properties of the spontaneous polarization in a ferroelectric thin film using the effective-field theory with correlations. The spin average profile in the ferroelectric thin film is nonuniform because of the surface parameter modification. The measured polarization in experiment is the mean value of the thin film [3]. Similarly, as a theoretical research, the spin average also refers to the mean value of the thin film. Usually, both the Curie temperature $T_{c}$ and the groundstate spin average $R(0)$ are increased with the increase of $J_{s}$ but decreased with the increase of $\Omega_{s}$. In this section, the four possible regions and the temperature dependence of spontaneous polarization will be numerically calculated from (5)-(15).

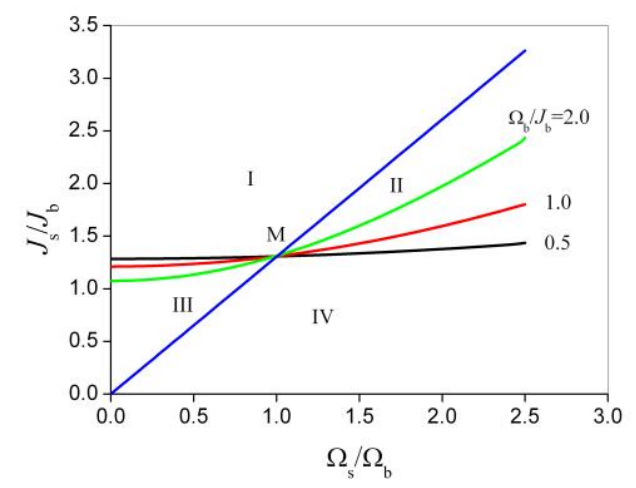

Figure 2. The four possible regions of the surface modification for the changes of the Curie temperature and the ground-state spin average.

In order to compare with the usual mean-field approximation, we follow the definition of $\mathrm{Qu}$ et al. in [3]. Fig .2 shows the phase diagram in the $\left(\Omega_{s} / \Omega_{b} \sim J_{s} / J_{b}\right)$ plane for different $\Omega_{b} / J_{b}$. It is obvious that the curves of $\Omega_{s} / \Omega_{b} \sim J_{s} / J_{b}$ depend sensitively on the bulk transverse field $\Omega_{b} / J_{b}$. Due to the surface modification, there exist four possible regions in Fig .2: region I, both $T_{c}$ and $R(0)$ enhanced; region II, $T_{c}$ enhanced but $R(0)$ reduced; region III, $T_{c}$ reduced but $R(0)$ enhanced; region IV, both $T_{c}$ and $R(0)$ reduced. The range of regions II and III decrease with increasing of the bulk transverse field $\Omega_{b} / J_{b}$. Meanwhile, a characteristic feature can also be observed, namely, the curves of $\Omega_{s} / \Omega_{b} \sim J_{s} / J_{b}$ for different bulk transverse field $\Omega_{b} / J_{b}$ intersect at a common intersection point $M$. Moreover, the abscissa value of $M$ lies on the line of $\Omega_{s} / \Omega_{b}=1.0$ which is independent of the layer number of thin film. To sum up, the behaviors for the changes of the Curie temperature and the ground-state spin average in the four possible regions of Fig .2 are similar to those of the usual mean-field approximation but different from the phenomenological theory [3].
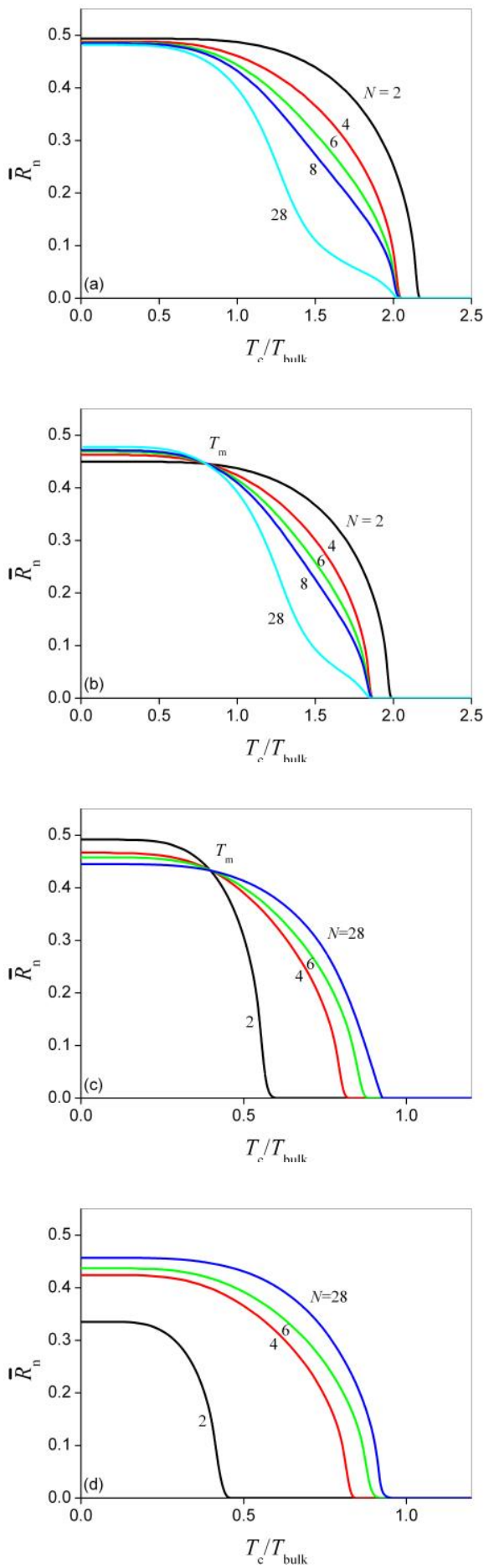

Figure 3. The temperature dependence of the spin average $\overline{R_{n}}$ corresponding to the four regions in Figure 2. (I) $J_{s} / J_{b}=3.0$, $\Omega_{s} / \Omega=1.0, \quad \Omega_{b} / J_{b}=1.0, \quad\left(\right.$ II) $J_{s} / J_{b}=3.0, \quad \Omega_{s} / \Omega=2.5$, $\Omega_{b} / J_{b}=1.0$, (III) $J_{s} / J_{b}=0.6, \Omega_{s} / \Omega=0.3, \Omega_{b} / J_{b}=1.2$, (IV) $J_{s} / J_{b}=0.6, \Omega_{s} / \Omega=1.0, \Omega_{b} / J_{b}=1.0$.

Fig .3 shows the Curie temperature dependence of the spin average for a thin film with different layer number $N$. It is obvious that the spin average $\overline{R_{n}}$ depends sensitively 
on the Curie temperature $T_{c} / T_{b u l k}$. The spin average $\overline{R_{n}}$ decreases with increasing of the Curie temperature $T_{c} / T_{b u l k}$. Corresponding to the four possible regions (I)-(IV) in Fig .2, there also exist four cases in Fig .3(a)-(d). It is obvious that Fig .3(a) and (d) show that the spin average of the thin film is larger or smaller than the bulk one unanimously, respectively. Meanwhile the Curie temperature, at which the spin average reduces to zero, is also higher or lower than the bulk one. However, the effects of layer number $N$ on the curves of $\overline{R_{n}} \sim T_{c} / T_{\text {bulk }}$ in Fig .3(a) and (d) are opposite to each other. These behaviors are similar to the usual mean-field approximation and the phenomenological theory [3]. On the other hand, there also exists a characteristic feature in Fig .3(b) and (c), namely the curves of $\overline{R_{n}} \sim T_{c} / T_{b u l k}$ intersect at a common Curie temperature $T_{m}$. Furthermore, the coordinate values of this crossover point are independent of the layer number $N$. On the left side of this crossover point, with increasing of the layer number $N$, the spin average increases. This is similar to that of the usual mean-field approximation but different from that of the phenomenological theory [3]. However, on the right side of this crossover point, with increasing of the layer number $N$, the spin average decreases. This is also in agreement with those of the usual mean-field approximation and the phenomenological theory [3].

As we all know, it has been proved that the usual mean-field approximation can give a qualitative description for phase transition behaviors of ferroelectric thin films by taking $J_{s}, \Omega_{s}, J_{b}, \Omega_{b}$ as the surface and bulk physical parameters. Usually, we may actually prefer to fit the usual mean-field approximation calculations to the experimental data in order to obtain the effects of surface modification on the Curie temperature and the spontaneous polarization. Similar to the usual mean-field approximation, the fitting is still not carried out and the dependence of the spontaneous polarization of TGS thin films on the Curie temperature [4] is also in agreement with Fig.3(b) calculated by using the effective-field theory with correlations.

\section{CONCLUSION}

In summary, within the framework of effective-field theory with correlations, the four possible phase transition regions in $\Omega_{s} / \Omega_{b} \sim J_{s} / J_{b}$ plane and the temperature dependence of spontaneous polarization have been investigated for an- $N$ layer ferroelectric thin film described by the transverse field Ising model. The numerical calculations show that the surface modification of exchange interactions and transverse fields might lead to the Curie temperature and the spin average increase or decrease in a different or same direction. Meanwhile, the results are similar to those of the usual mean-field approximation, but different from the Landau phenomenological theory to some extent. Furthermore, the effective-field theory with correlations can also reasonably describe the temperature dependence of the spontaneous polarization in experimental facts of triglycine sulfate (TGS) thin films.

\section{REFERENCES}

[1] J. F. Scott, "Applications of modern ferroelectrics," Science,vol. 315, Feb. 2007, pp. 954-959, doi: 10.1126/science.1129564.

[2] M. Dawber, K. M. Rabe, and J. F. Scott, "Physics of thin-film ferroelectric oxides," Rev. Mod. Phys., vol. 77, Oct. 2005, pp. 1083-1130, doi: 10.1103/RevModPhys.77.1083.

[3] B. D. Qu, W. L. Zhong, and P. L. Zhang, "Phase-transition behavior of the spontaneous polarization and susceptibility of ferroelectric thin film," Phys. Rev. B, vol. 52, Jul. 1995, pp. 766770, doi: 10.1103/PhysRevB.52.766.

[4] A. Hadni and R. Thomas, "High electric fields and surface layers in very thin single crystal plates of triglycine sulfate," Ferroelectrics, vol. 59, Mar. 1984, pp. 221-232, doi 10.1080/00150198408240092.

[5] W. L. Zhong, Y. G. Wang, P. L. Zhang and B. D. Qu, "Phenomenological study of the size effect on phase transitions in ferroelectric particles," Phys. Rev. B, vol. 50, Jul. 1994, pp. 698 703, doi: 10.1103/PhysRevB.50.698.

[6] C. L. Wang, W. L. Zhong, and P. L. Zhang, "The Curie temperature of ultra-thin ferroelectric films," J. Phys.: Condens. Matter, vol. 4, May 1992, pp. 4743-4749, doi: 10.1088/09538984/4/19/014.

[7] H. K. Sy, "Surface modification in ferroelectric transitions," J. Phys.: Condens. Matter, vol. 5, Mar. 1993, pp. 1213-1220, doi: 10.1088/0953-8984/5/9/006.

[8] X. G. Wang, S. H. Pan, and G. Z. Yang, "Effects of multi-surface modification on Curie temperature of ferroelectric films,", J. Phys.: Condens. Matter, vol. 11, Aug. 1999, pp. 6581-6588, doi: 10.1088/0953-8984/11/34/310.

[9] C. L. Wang, S. R. P. Smith, and D. R. Tilley, "Ferroelectric thin films described by an Ising model in a transverse field," J. Phys.: Condens. Matter, vol. 6, Nov. 1994, pp. 9633-9646, doi: 10.1088/0953-8984/6/45/013.

[10] X. Z. Wang, X. Y. Jiao, and J. J. Wang, "Magnetic properties of a transverse Ising film with $s=1 / 2$," J. Phys.: Condens. Matter, vol. 4 , Mar. 1992, pp. 3651-3658, doi: 10.1088/0953-8984/4/13/024.

[11] B. H. Teng and H. K. Sy, "Parameter modifications in the phase diagrams of the transverse field Ising model," Physica B, vol. 348, May 2004, pp. 485-492, doi: 10.1016/j.physb.2004.02.006. 